\title{
EDUCACIÓN PÚBLICA Y COVID-19: CUANDO PENSAR FUERA DE LA CAJA ES DE VIDA O MUERTE
}

Victor Orellana Calderón e Iván Salinas Barrios 


\section{VÍCTOR ORELLANA CALDERÓN}

Sociólogo de la Universidad de Chile, Magíster y Doctor (c) en Ciencias Sociales de la misma Casa de Estudios. Es Investigador Asistente del Centro de Investigación Avanzada en Educación (Ciae) de la Universidad de Chile. Sus líneas de investigación son la sociología de la educación y la estructura social.

\section{IVÁN SALINAS BARRIOS}

Químico de la Universidad de Chile, Ph.D en Teaching and Teacher Education de la Universidad de Arizona. Profesor Asistente del Departamento de Estudios Pedagógicos de la Universidad de Chile. Sus líneas de investigación son la educación en ciencias y la formación docente. 


\section{EDUCACIÓN PÚBLICA Y COVID-19: CUANDO PENSAR FUERA DE LA CAJA ES DE VIDA O MUERTE}

\section{LA EDUCACIÓN ENTRE EL FRENO Y EL ACELERADOR, SIN PODER VER EL VOLANTE}

La pandemia del Covid-19 tomó a todo el mundo por sorpresa. Aunque la reacción de los Estados y las instituciones ha sido dispar, lo cierto es que el Coronavirus ha sido un recordatorio de las tareas pendientes de nuestra civilización —a pesar de todo su poderío tecnológico y económico-.

No es solo el factor sorpresa el que ha facilitado la expansión del Covid-19. Esta pandemia aparece en un mundo mucho más interconectado, lo que facilita su rápida expansión global. Irónicamente, esa interconexión viene de la mano, en la hegemonía neoliberal, del desmantelamiento de las instituciones solidarias edificadas en el siglo pasado, aquellas que, ante las incertidumbres provenientes del mercado o de la naturaleza, respondían como acción colectiva consciente y como soporte social, cultural e institucional.

Chile no ha estado ajeno a esta contradictoria realidad. Sus autoridades de gobierno han destacado por su errático manejo de la pandemia, lo que lo ha convertido en uno de los países con más contagiados en relación a su población ${ }^{1}$. Y aunque cabe en ello responsabilidad a las autoridades de turno, lo cierto es que las instituciones de solidaridad y corresponsabilidad en el país han sido desmanteladas hasta en su dimensión más básica en las pasadas décadas. Con escasa salud y educación públicas y con un Estado desvinculado de los servicios básicos como el alcantarillado, la electricidad y el acceso a Internet, las autoridades del país cuentan con mínimos instrumentos para coordinar una respuesta coherente, global y eficiente ante la crisis. Por lo mismo, y compelidos a hacer frente a la situación con la misma soledad con que han debido abrirse paso en la vida económica y social, los chilenos echan mano a sus recursos individuales y familiares para protegerse. Con ello queda a la vista nuestra triste desigualdad y reaparece el hambre en contextos de pobreza urbana. En suma, la fragmentación de la sociedad queda sin velos.

1. Ver nota de Radio Bío Bío, 26 de mayo de 2020. https:/ /www.biobiochile.cl/noticias/nacional/ chile/2020/05/26/chile-supero-paises-mas-golpeados-pandemia-casos-diarios-millonhabitantes.shtml 
El desconcierto ha sido particularmente agudo en dos instituciones clave para la edificación de la vida moderna: la salud y la educación. Aunque existen todavía rémoras institucionales de los intentos previos del país por forjar sistemas modernos de carácter público, el grueso de su expansión reciente ha corrido de la mano de los mercados, sin instancias de coordinación sistémica. Tal ausencia de planificación y coordinación ha sido divulgada como virtud en la medida en que su construcción emerge de las elecciones individuales ante la oferta de mercado en lugar de una razón de Estado (Beyer, 2001). Más aún, el propio Estado ha tendido a sumarse a este coro de instancias particulares, debilitando - o eliminando totalmente, como ocurre en la educación- lógicas de articulación sistémica. El Estado se ha desplegado más como apoyo financiero a los particulares y como fuente de equivalencias técnicas para que el mercado intercambie racionalmente servicios por dinero — de ahí su obsesión con la medición de logros y el discurso de la calidad-, renunciando a una dirección y articulación de conjunto de estos ámbitos.

Una escena icónica del desconcierto fue la polémica que los ministros de las carteras de Salud y Educación tuvieron a fines de abril de 2020 — prensa incluida ${ }^{2}$ a raíz de la suspensión de clases en escuelas y liceos. Mientras el ministro de Salud calificó la medida como un error, argumentando que los y las niños/as habían quedado a la deriva, sin la protección y el respaldo que les da la escuela, el ministro de Educación defendió la suspensión de clases amparado en un amplio respaldo médico, científico y de los propios actores de la educación, en especial del Colegio de Profesores, pues las escuelas son un espacio de socialización e interacción que multiplica los contagios (de cualquier enfermedad infecciosa).

En las posiciones de ambos secretarios de Estado despunta, de cierto modo, el debate sobre la educación en las primeras semanas de la pandemia.

De un lado se postuló que la educación no podía detenerse, posición que se justificó en dos argumentos principales: el primero, relacionado con el desempeño (hay contenidos que son demasiado relevantes para la formación de los sujetos), y el segundo, con la equidad (si se abandona a los más pobres, los ricos acrecentarán su conocida ventaja educativa). Las autoridades de gobierno rápidamente adoptaron este discurso, haciendo hincapié en los recursos digitales, y aceleraron la llegada a la educación del teletrabajo. Ello terminó por hacer más nítidas las dificultades de una sociedad tan desigual como la chilena, cuyos recursos tecnológicos y de acceso a Internet distan de estar igualmente distribuidos. La máxima, independientemente

2. Ver nota de El Mostrador, 21 de abril de 2020. https://www.elmostrador.cl/noticias/ 2020/04/21/mineduc-le-responde-a-manalich-ministro-figueroa-aseguro-que-la-decision-decerrar-los-colegios-se-tomo-escuchando-a-todos-los-actores/ 
de los recursos tecnológicos, era adaptar a la pandemia las prácticas educativas existentes. Esto explica la decisión inicial de mantener el Simce en 2020, aunque declaradamente sin consecuencias para las escuelas ${ }^{3}$.

De otro lado se ha puntualizado en la excepcionalidad del año académico 2020 producto del Covid-19, y a raíz de ello se ha sugerido eliminar y/o menguar prácticas del sistema educativo que resulten agobiantes para los sujetos — directivos escolares, profesoras y profesores, estudiantes y sus familias y cuidadores- y que incrementen el estrés en esta situación crítica. En la medida en que la educación chilena ya arrastraba un malestar previo, las medidas propuestas tendían a ser la suspensión, flexibilización o derechamente eliminación de determinados procedimientos cargados de cierta polémica. Así, se ha propuesto la suspensión del Simce, de la evaluación docente ${ }^{4}$, de los pagos y, recientemente, se ha sugerido que los estudiantes pasen de curso de forma automática (Bellei y Muñoz, 2020).

Tras este debate educativo corría también un debate económico y político. En la medida en que las estructuras de financiamiento de la educación chilena se organizan en lo medular a partir de precios unitarios por estudiante - pagos directos, subvenciones, vouchers o créditos-, suspender las actividades tiene el necesario efecto de disminuir los recursos de las instituciones. Lo que es obvio en el caso de una compraventa privada - que no tiene sentido pagar por un servicio que no se entrega- ocurre también con el Estado. Como hemos discutido antes (Orellana et al., 2018), todo sistema de voucher tiene un mecanismo de comprobación del servicio en la medida en que es un pago unitario (compra) y no un aporte institucional. De ahí que un argumento implícito para sostener las actividades educativas se ancla en el simple hecho de que el pago es por asistencia en el ámbito escolar y a través de la demostración de la condición de estudiante regular cuando hablamos del pago del Crédito con Aval del Estado y la gratuidad en el nivel superior (dos o más veces al año).

Aunque resquicios legales mantienen hasta el momento la entrega de recursos públicos a nivel escolar (que dependen de la asistencia), en el nivel superior el escenario es más complicado dado el mayor volumen de los pagos privados y la

3. Ver nota de Radio U. de Chile, 12 de mayo de 2020. https://radio.uchile cl/2020/05/12/ mineduc-confirma-simce-2020-tendra-un-caracter-de-diagnostico-frente-a-la-pandemia/

4. Ver nota de Radio Cooperativa, 5 de mayo de 2020. https://www.cooperativa.cl/noticias/ sociedad/salud/coronavirus/mesa-social-covid-19-propuso-eliminar-las-repitenciasescolares-por-este/2020-05-05/072047.html. Ver informe de Educación Mesa Social Covid-19. https://www.uchile.cl/documentos/propuestas-sobre-educacion-mesa-socialcovid-19_163074_0_4342.pdf https://www.cooperativa.cl/noticias/pais/educacion/colegios/ colegio-de-profesores-pide-aplicar-ya-propuestas-de-la-mesa-social/2020-05-05/145209.html 
tendencia de los estudiantes a abandonar y/o congelar sus estudios. No poder sostener las actividades regulares para las instituciones de educación superior dista entonces de ser un debate académico o de salud: se trata de su supervivencia, sobre todo cuando en los años previos la expansión de la matrícula se ha detenido y, peor, ha comenzado su caída en algunas instituciones y carreras. No es de extrañar, entonces, el entusiasmo que despierta hoy la gratuidad en instituciones privadas vinculadas a o de propiedad de antes enérgicos opositores a la idea de la educación superior gratuita ${ }^{5}$.

Es así como las distintas posiciones escépticas del confinamiento —en educación y en el ámbito laboral - terminan siendo afines, pues consideran a las escuelas, liceos e instituciones terciarias como un ámbito más de la economía. De hecho, en términos agregados, el peso de la educación llegó al 9 por ciento del PIB en 2016 (Ocde, 2019). La educación se contemplaba como parte de la "nueva normalidad" en los planes del gobierno durante mayo de 2020. Mantener las actividades económicas implica mantener las ventas, lo que sostiene el flujo y los empleos asociados. De esto se sigue que el apoyo estatal ha de dirigirse a asegurar las ventas, léase, los ingresos de las instituciones de educación, para que puedan seguir haciendo lo que saben hacer: educar. En esa línea ha estado el planteamiento de Carlos Williamson - Rector de la Universidad San Sebastián, propiedad de la familia Cueto y Navarro, entre otros- y de sendos personeros de la educación privada, que solicitan acelerar la aprobación del nuevo crédito de reemplazo al CAE o bien crear uno nuevo de emergencia ${ }^{6}$. En esa misma dirección se ha movido el gobierno con sus anuncios de protección a la clase media ${ }^{7}$.

Puesto el asunto de la educación en el contexto de la economía, es natural que las diferencias sociales se manifiesten. El debate hasta acá ilustrado se aplica únicamente donde cabe responsabilidad estatal asociada a las prestaciones que compra el Estado a privados y a sí mismo. Tanto la educación escolar particular pagada como las universidades más elitistas descansan en la capacidad de sus clientes y la espalda financiera de sus grupos controladores y sostenedores, casi como si habitaran un país distinto. Paradojalmente, los segmentos acomodados, que

5. Ver nota de El Mostrador, 18 de mayo de 2020. https://www.elmostrador.cl/noticias/ pais/2020/05/18/efecto-coronavirus-otros-cinco-planteles-de-educacion-superior-pidensumarse-a-la-gratuidad/

6. Ver nota de El Mostrador, 18 de mayo de 2020. https://www.elmostrador.cl/noticias/ pais/2020/05/18/las-opciones-desalvataje-al-sistema-de-educacion-superior-ante-la-crisisfinanciera-que-genero-el-coronavirus/

7. Ver nota de 24 Horas, 22 de mayo de 2020. https://www.24horas.cl/tesirve/clase-mediaprotegida-conoce-todos-los-beneficios-del-programa-de-proteccion-social-3326166 
importaron el virus en la sociedad por su mayor contacto físico con el extranjero, hoy son los más guarecidos contra la pandemia. Pero incluso acá, por la recesión, la parte recién avenida a estos sectores ha tenido dificultades económicas, lo que evoca ese divulgado gesto de grupos emergentes en la crisis asiática del 98, que tuvo que abandonar el espacio de la educación privada para retornar a la subvencionada.

En suma, tanto la inercia del desempeño académico como el económico llaman a sostener, hasta el punto en que sea posible, las actividades educativas. Por mientras, las voces críticas - de los actores sociales y de las y los investigadores y académicos del área- han puesto el énfasis en la suspensión y/o flexibilización de los contenidos y prácticas de la docencia. En este escenario, las diferencias sociales han tendido a acrecentarse, en tanto la desigualdad establece sectores con problemáticas totalmente distintas a la hora de enfrentar la crisis, casi como si fuesen sociedades distintas. La educación chilena se asemeja a un vehículo que, de frenar, aumenta las desigualdades y se desploma económicamente; y de acelerar, también las acrecienta, con el agregado que su sobrevida económica, enlazada a determinada clase de prácticas, termina agobiando aún más a las personas en una situación ya tremendamente estresante.

Aunque estas dos posiciones - ilustradas acá como el freno y el acelerador- han dominado el debate de los últimos meses, no han sido las únicas. La propia Mesa Social y su subcomisión sobre educación convocada por el gobierno en la materia — dirigida por prestigiosos académicos de las principales universidades del paísincluía en las páginas de su informe algunos puntos que escapaban a estos dos polos. Aun tratándose de afirmaciones laterales a las ideas fuerza del documento -en la línea de las autoridades gubernativas-, el texto puntualiza que un papel clave de la educación es la construcción del bienestar socioemocional de los estudiantes, condición de posibilidad para cualquier aprendizaje (Varios autores, 2020).

Independientemente del modo en que haya sido planteado - limitado a una forma de bienestar individual y no a un papel de construcción social y nacional-, este punto marca el hecho de que la educación no solo ha de dejar de agobiar a las personas, sino que debe ser fundamentalmente un apoyo. Esto permite recuperar la idea original de la educación como ejercicio de construcción consciente de la vida social e individual, de sus fundamentos o condiciones culturales de posibilidad. En nuestra metáfora del vehículo, este tipo de ideas nos recuerda que la educación no tiene solo freno y acelerador, sino que su sentido es también nuestra responsabilidad: la educación tiene volante o dirección. Para la educación clásica, la idea de igualdad siempre apuntó a la construcción nacional, a la construcción de la comunalidad entre las distintas partes de la sociedad en tanto espacio de convivencia política y no de competencia individual vía agregación de capital humano. En varios países 
del mundo estas ideas han estado presentes y han sido centrales en la respuesta de la educación a la pandemia ${ }^{8}$.

Las dificultades de la educación chilena frente al Coronavirus no solo están relacionadas con un déficit de gestión de las actuales autoridades. Ciertamente, influyen instituciones que, fundadas para fiscalizar y optimizar la educación, se han transformado - en su efecto conjunto- en parte del problema. Al edificar la educación como enjambre de instituciones particulares enraizadas cada cual con su clase social correspondiente, y arrastrado el Estado a ese caos segregado bajo una manipulada retórica del capital humano, la competencia, la calidad y el management, se da la ironía de que, en un momento de crisis, lo que se supone un respaldo para la sociedad se transforma en una fuente más de incertidumbre.

\section{LAS RAÍCES DEL DESCONCIERTO EDUCATIVO}

¿Por qué hemos llegado a este punto en que pareciera que lo mejor que puede hacer la educación es detenerse? Si descartamos el déficit de gestión gubernativa como razón principal, hemos de mirar entonces el proceso histórico inmediato. Las estructuras del agobio en la educación, por llamarles de algún modo, no forman parte del diseño original de la reforma de la dictadura y no han aparecido de manera espontánea desde el mercado. Irónicamente, todas ellas — desde el ensamble del Simce con el financiamiento a las escuelas, la evaluación docente y la fijación de precios en educación superior para el CAE y luego para la gratuidad- han sido responsabilidad de la acción estatal durante los gobiernos civiles de la postdictadura. Estas instituciones se han propuesto, de hecho, introducir elementos racionales y de equidad al mercado. Reconstruyamos sucintamente este proceso.

El desarrollo educativo durante la década de los ochenta fue escaso, pues se construyeron las condiciones institucionales para su mercantilización. Este proceso se encontraba solo parcialmente desarrollado en 1990: todavía el grueso de la matrícula en los niveles escolar y superior era estatal y aún estaban pendientes, en lo medular, los desafíos civilizatorios de mediados del siglo XX; a saber, la edificación de un sistema de educación moderno y universal.

En el inicio de la democracia, conscientemente, las nuevas autoridades evitaron hacer reformas estructurales (Cox, 1994). Intentaron acometer las tareas de envergadura civilizacional impulsando el modelo heredado en lugar de revertirlo.

8. Ver el sitio web de emergencia creado por Unesco para la orientación de los sistemas educacionales en el mundo ante la amenaza del Covid-19. https://es.unesco.org/covid19/ educationresponse 
En ello, fueron configurando la acción estatal bajo la base de los programas, esto es, políticas relativamente livianas y focalizadas de ajuste a la institucionalidad previa. Más adelante, como reacción a las masivas movilizaciones de estudiantes del 2006, se construirían las bases argumentales y políticas para crear instituciones propias de los mercados de servicios sociales regulados. Entonces aparecen las superintendencias o las agencias de calidad, que no fueron creadas en dictadura, tanto por el escaso desarrollo del sector privado como porque la dictadura tenía en educación una idea más de libre mercado que de uno regulado. Esto sería notorio sobre todo en el peso perentorio que se le dio más adelante a la calidad medida por el Estado, por encima de la calidad que los propios clientes atribuían a la educación. En suma, la acción estatal iría, capa por capa, agregando instancias a una estructura de financiamiento y competencia mercantil previamente establecida. Son estas capas, sean programas o instancias reguladoras y técnicas, las que más adelante serán llamadas, en genérico, políticas públicas o, simplemente, políticas educativas.

Esta estrategia logró una expansión rápida de la educación combinando aportes estatales con un gasto privado cada vez mayor por parte de las familias (como ocurrió con el copago instaurado en 1993 en el nivel escolar y el CAE en la educación superior). El problema de esta estrategia fue que nunca alcanzó el desempeño académico que se esperaba y, aparte, se amoldó con mucha facilidad a la ya aguda desigualdad de nuestro país (Guajardo y Jara, 2018; Salazar y Leihy, 2013). Tras la reforma de 1994, los resultados no mejoraron como se deseaba (Acuña, 2015) y el crecimiento en la educación superior no se centró en las instituciones de mayor calidad, sino todo lo contrario. Desde el año 2000 se hizo patente la segregación tanto en el nivel escolar como superior (Redondo, Descouvières y Rojas, 2004). Esto era doblemente problemático, como demostraron las movilizaciones de 2006 y 2011, dado que la educación misma había pasado de ser la base sociocultural de la nación a ser considerada una "cantera" de capital humano para estrategias individuales de movilidad social. Es decir, si la educación no agregaba capital humano como debía, no solo la educación peligraba, sino también la movilidad social, y con ella, la idea de democratización social formulada en la transición política (Ruiz Encina, 2015).

Con una educación expansiva pero que no alcanzaba el estándar deseado ni en equidad ni en calidad, la respuesta del régimen fue, básicamente, tensar sus políticas para forzar al mercado a ser un poco más equitativo y eficaz. Por supuesto, la equidad se entendía como una menor segregación dentro de la población que asistía a instituciones subvencionadas por el Estado. Así, la superación de la ruptura social entre la mayoría del país y los sectores altos (parapetados en la educación elitaria 
pagada que no recibe subvenciones) quedaría como una quimera tipo Machuca ${ }^{9}$, propia del idealismo de los sesenta y setenta. El Estado incrementó entonces los efectos performativos de sus políticas y su fuerza coactiva sobre las prácticas de los sujetos, encontrando en el financiamiento estatal su gran palanca de presión.

Este esquema profundizó el abismo entre la educación mayoritaria y la elitaria, pero, además, tensó la elaboración de política, pues fue necesario buscar nuevas formas de optimización y equidad. El campo de la investigación en educación se transformó en la fuente decisional en este proceso, puesto como base para la política basada en evidencia. Con el tiempo, los investigadores que buscaban respuestas para estas preguntas - que eran bien específicas y propias de las decisiones anteriorescrearon un circuito cada vez más activo con las autoridades ministeriales, los parlamentarios - cuando se requerían ajustes legales-y luego con los intereses privados en educación, que ya durante los dos mil se hicieron de la mayor parte de la matrícula. Este campo no carecía de conflicto en términos de las soluciones que buscaba para sus materias, con voces más o menos preocupadas por la equidad y la inclusión, más o menos preocupadas por la calidad y otras más o menos preocupadas por la libertad de las familias de elegir sus opciones. Pero, con todo, su prolífica producción y debate convergía en lo medular: la reducción de la política educativa al ajuste técnico de la educación existente.

En el caso escolar, la presión se concentraría en los profesores, responsabilizados por una educación que no despuntaba a pesar de las reformas hechas, ya fuera por la falta de política estatal en la materia o un alegado déficit de desempeño (Bellei, 2001; Mizala y Romaguera, 2003). Es entonces - y no en dictadura- que las pruebas del Simce comienzan a vincularse con una serie de aspectos financieros y de evaluación docente (Sistema de Evaluación Nacional de Evaluación del Desempeño, Sned). Esto somete la entrega de porciones crecientes de recursos a las escuelas y también del salario de los profesores a los resultados ordenados como competencia. Con el tiempo, las escuelas y liceos girarán del currículum declarado al verdadero currículum fáctico de la educación chilena: el Simce.

Luego, como respuesta a la movilización de 2006, el fin formal de la Loce pero no de la arquitectura básica de la educación chilena-implicó la construcción de todo un Sistema de Aseguramiento de la Calidad (el SAC, liderado por la Agencia de Calidad), también basado en el puntaje Simce. Este esquema contempló el cierre de escuelas en determinados casos de bajo desempeño, lo que, como resulta obvio, concentró la amenaza en las escuelas más pobres y en las escuelas públicas. Para

9. La película de Andrés Wood (2004) retrata los conflictos que suscita el intento de terminar con este tipo de segregación educativa en los años de la Unidad Popular. 
promover mayor equidad en el sistema, se aumentó dramáticamente la subvención a los estudiantes más pobres con la Ley de Subvención Escolar Preferencial (SEP), lo que traería como consecuencia una disminución aún más acelerada de la educación estatal (municipal) en aras de la mayor rentabilidad que ahora significaban los estudiantes pobres para los colegios privados con financiamiento público (particularsubvencionados).

En educación superior, el equivalente al ensamble que condiciona el aumento de recursos a las evaluaciones de desempeño (Simce-SAC-Sned-SEP) fue el arreglo CNA-CAE. Este juntó el Sistema de Aseguramiento de la Calidad con la entrega de recursos vía Crédito con Aval del Estado (CAE). Aunque la educación superior ya se expandía rápidamente en la primera mitad de los dos mil, su crecimiento adolecía de dos patrones cuestionables: se desplazaba desde las capas más acomodadas a sus entornos adyacentes, lo que impedía la incorporación de los segmentos más pobres (Orellana 2011) y, al mismo tiempo, no parecía dar lugar a una calidad satisfactoria. La aproximación de las políticas fue corregir esta tendencia del mercado -intelectualmente elaborada como el papel del Estado de guiar al mercado (Brunner et al., 2005) - inyectando más recursos al sistema, focalizados en los segmentos más bajos, mientras se depositaba en la acreditación institucional la llave para el acceso a estos aportes.

Tras 2011, a raíz de las movilizaciones sociales, esta dirección de marcha podría haber sido alterada. No obstante, se mantuvo. La reforma del segundo gobierno de Michelle Bachelet más bien trató de tomar partido al interior del debate de la política pública existente. El gobierno convocó a quienes eran partidarios de aumentar las exigencias al mercado y, por lo mismo, de una mayor drasticidad estatal (pero no del Estado en general, sino de esta forma específica de Estado que venía construyéndose). Así, se sumó una nueva capa de exigencias estatales a la ya edificadas en los tres lustros anteriores, lo que condicionó un nuevo aumento en la entrega de recursos, ahora ya a una educación de matrícula mayoritaria y nítidamente privatizada.

En otros lugares se han discutido con más detalle los límites y alcances de esta reforma (Huneeus, 2016; Salazar, 2015; Sanhueza y Orellana, 2018). Lo que interesa aquí son las consecuencias de largo plazo de un curso ininterrumpido de marcha - la mercantilización educativa - en tres ámbitos distintos: los actores, la política y la ciencia.

Para los actores —estudiantes y sus familias, profesores, académicos, trabajadores e instituciones-, la lenta acumulación de estas políticas ha significado una estandarización y cuantificación burocrática cada vez más aguda de su trabajo, lo que ha estimulado en ellos conductas más instrumentales que sustantivas. La evidencia en este sentido es abundante (Parcerisa y Falabella, 2017; Ruiz Schneider, Reyes y Herrera, 2019; Fardella y Sisto, 2015; Fardella, 2016). En la medida en que tales 
métricas se suman unas con otras (no hay elaboración de su efecto agregado pues se diseñan por partes), cuantificando resultados de procesos que son enormemente complejos, tienden a percibirse como exigencias de poca utilidad práctica a la vez que perentorias. Los sujetos experimentan un quiebre en su tiempo de trabajo, a saber, las horas dedicadas a su labor en sí, y las horas dedicadas a alcanzar las métricas - puntajes, proyectos adjudicados, años de acreditación, artículos indexados, etcVale la pena apuntar un resultado no buscado de este tipo de prácticas cuando se aplican con criterios de equidad: la cuantificación de la pobreza termina en un sello que estigmatiza a los sujetos. Peor aún, los planes de mejora tienden a agregarse a las horas regulares de trabajo, lo que genera mayor agobio, como se ha registrado en los programas de acompañamiento a los estudiantes pobres en educación superior (Quintela Dávila, 2013; Orellana et al., 2019).

La reducción del debate educativo a la política educativa, puesta en estos términos, naturalmente tuvo un efecto en la política en general. El sentido de marcha global de la educación, paradojalmente, queda excluido de la política. Luego la política tiende a pensarse como la gestión y ajuste de la regulación de mercado. Si a inicios de los noventa mantener el modelo fue una decisión consciente, en lo sucesivo ya no: ajustar y podar el modelo se naturalizó y se hizo todo el horizonte posible de la acción, incluso del debate entre izquierda y derecha. La obsesión por la calidad centraba la mirada en cada institución por separado, como si fuesen fábricas de rendimientos y, luego, la mejora se entendió como la resiliencia y capacidad de dichas unidades de sobreponerse a la adversidad (Bellei et al., 2014). Ya ni siquiera sería posible imaginar algo distinto.

En estricto rigor, en el periodo hay poca reflexión política sobre la educación, pues su organización básica y su sentido están naturalizados. Existe, sí, mucho debate sobre qué le corresponde hacer al Estado. Las leyes, por ejemplo, dejan de ser grandes organizadoras de sentido y más bien se elaboran como sistemas de tarificación y/o cuantificación del desempeño. Esto aumenta la complejidad —en apariencia-, puesto que la discusión pública se ve inundada de un enjambre de ajustes parciales con intrincadas siglas, indicadores y lenguajes de expertos, y se vacía de un sentido de dirección general comprensible para el debate ciudadano. De ahí que, en los últimos lustros, en pleno régimen de democracia formal, las capas tecnocráticas más bien hayan aumentado su poder y figuración en lugar de disminuirlos. Su cercanía con la investigación en educación, de un lado, y con las burocracias políticas, por otro, le ha permitido a la tecnocracia virtualmente monopolizar la acción estatal en educación. Por eso, cuando aparecen las protestas de 2006 y 2011 (que toman por sorpresa a este campo académico y su tecnocracia adyacente), el Estado no puede ver en la sociedad "interlocutores racionales". Si no se es parte de la racionalidad tecnocrática, se carece entonces de razón (Figueroa, 
2012; Domedel y Peña y Lillo, 2008). Hay que relevar cuánto de la propia proyección material de la tecnocracia requiere de este arreglo y cómo ese papel deviene un modo de vida. Los ingresos de la tecnocracia son notoriamente más altos que los de los profesionales asalariados y los académicos, integrando sueldos universitarios con proyectos y/o salarios exclusivos de alta dirección (pública o privada). Es bajo este peso tanto ideológico como material que la acción estatal decanta, se consolida y se hace sorda a las crecientes protestas sociales, incluido aquí el estallido de octubre de 2019 (Ruiz Encina, 2020).

Finalmente, la tecnocracia también tiene un efecto sobre la ciencia. Aparte de cerrar el debate político, cerca la investigación científica propiamente tal. Bajo la máxima de política basada en evidencia, en las últimas décadas la investigación en educación ha sufrido una fuerte constricción en sus preguntas potenciales y medios para responderlas. En aras de un supuesto compromiso con el conocimiento útil y concreto, por oposición a la filosofía educativa especulativa y abstracta, lo cierto es que se ha reducido la investigación a una aproximación gerencial sobre los modos en que cada unidad educativa aislada —entendida como fábrica de rendimientospuede aumentar sus desempeños en los instrumentos arriba comentados o bien integrar diferencias sociales entendidas como rasgos cuantificables de individuos (vulnerables, de necesidades educativas especiales, etc.).

Esta aproximación no releva lo concreto, sino que, basada en tal retórica, más bien desatiende el vínculo entre educación, sociedad e individuo. Así, la observación de la educación en lo macro y lo micro queda condicionada a preguntas y encuadres epistemológicos, teóricos y metodológicos prefigurados; por ello el malestar educativo es invisible y se manifiesta siempre como sorpresa ante este campo académico. Apelando al conocimiento concreto, la investigación comporta una fuerte carga abstracta y normativa - cuando no ideológica- con conceptos como calidad, mejora y liderazgo. Estos conceptos dan lugar a una gramática que oscurece los dilemas pedagógicos y políticos concretos de la educación.

Es una investigación, en suma, más bien dirigida al ajuste del mercado que a construir conocimiento científico sobre la educación como proceso social complejo. La elaboración de hipótesis, por ejemplo, donde la filosofía de la ciencia sugiere que se juega la mayor cuota de imaginación en la investigación (Molina, 2006; Lakatos, 1989), acá viene ya prefigurada por las políticas y sus ajustes posibles. De una ciencia indagatoria se pasa a un enfoque ingenieril, pues de lo que se trata es de optimizar desempeños (académicos o en equidad) y no de comprender el proceso educativo en su relación con el devenir de la sociedad y la cultura. Así se termina en una inercia investigativa de escaso desarrollo pedagógico y precaria imaginación científica.

Es cierto que el papel del Estado en las últimas tres décadas ha permitido alcanzar una educación de cobertura universal casi en todos los niveles. Pero este 
avance nos ha llevado a un desarrollo de las instituciones y la acción estatal que, en un contexto de mercado, ha presionado a los actores a un comportamiento cada vez más instrumental y agobiante. A medida que la educación se torna fundamental para que las personas y sus familias enfrenten el mercado laboral (por el proceso general de credencialización que experimenta la sociedad chilena), la educación formal y su agobio ocupan cada vez más horas del día y años de la vida.

Mientras, una reducida élite se separa de la educación mayoritaria subvencionada (estatal y privada) por medio de ambientes educativos altamente segregados. Este problema, a pesar de todo lo que se ha discutido sobre equidad, sigue siendo considerado como inevitable. Irónicamente, en los ambientes elitarios no se trabaja para los desempeños medidos como objetivo central -a no ser que se busque validación frente a la educación tradicional-, sino que la enseñanza se ancla en proyectos más bien ideológicos con cierres sociales muy fuertes. Ser parte de un segmento alto se expresa, paradojalmente, en la capacidad de escapar del agobio inconducente de la educación subvencionada para poder centrarse entonces en objetivos pedagógicos y de carácter sociocultural y comunitario (Bellei, Orellana y Canales, 2019; Madrid, 2016). En una frase, los ricos escapan del mercado de masas costeando proyectos comunitarios exclusivos, impermeables a los tentáculos estatales de agobio creados en las últimas dos décadas.

\section{EDUCACIÓN Y PANDEMIA: MIRAR EL PRESENTE DESDE EL FUTURO}

Hecho este recorrido, tiene sentido volver la mirada sobre la coyuntura. El debate entre el freno y el acelerador y el desconcierto a que nos arrastra encuentra sus raíces en un enjambre de instituciones y acción estatal que ha reducido la educación a la producción de desempeños individuales como condición para pagos también individuales. La mirada ha estado centrada en optimizar partes, haciendo invisible la totalidad. En su movimiento conjunto, las distintas medidas y exigencias derivan en desempeños u obligaciones de pago que generan agobio y segregación; de ahí que la demanda inmediata de los actores sea detenerlas. Pero luego, como de los mismos desempeños y pagos depende el andamiaje general de la educación (centralmente su flujo financiero y, con ello, sus empleos), las autoridades insisten en mantenerlos con la misma energía con que han insistido en mantener la actividad económica durante la pandemia.

Puesto de este modo el problema ante la emergencia sanitaria, los dos polos del debate son inconducentes y ninguno entrega las claves para redirigir los esfuerzos educativos en la pandemia. Por cierto, el agobio debe parar, pero detenerse y/o flexibilizarse no basta para que la educación responda a la demanda que la coyuntura 
instala. Del otro lado, tampoco satisface las necesidades de la coyuntura seguir su calendario, sea presencial o por Internet. El problema no está en los recursos digitales o en la educación a distancia. El problema está en los objetivos. Pero antes de revisar cuáles pueden ser estos objetivos y los medios para alcanzarlos, cabe un comentario sobre el esfuerzo político e intelectual que resulta necesario para definirlos.

Decíamos que la política educativa basada en evidencia, irónicamente, terminó cercando tanto el debate político como a la ciencia y su aporte a la educación. Esto no debe llevar a una ciencia sin interés por la práctica o a un idealismo político sin sustento. Por el contrario, debe llevarnos a recuperar una aproximación propiamente racional. La política, y para esto no hay que ir más allá del ideario liberal, no puede tener determinaciones a priori (Bobbio, 1989; Weber, 2005; Arendt, 1997), ni siquiera las que brinde la evidencia en determinado momento histórico. La técnica puede apuntalar los medios de la educación, pero no los fines. Las políticas no se basan en evidencia; son algunos de sus instrumentos los que requieren del apoyo de la ciencia y la técnica. No hay, en definitiva, ni argumento científico ni técnico que permita dirimir la pugna entre sistemas de mercado o públicos: esta discusión es eminentemente política, por cuanto sus diferencias remiten a proyectos de sociedad y no a brechas de desempeño.

Es decir, en educación cabe a la ciencia y la técnica un papel subordinado a la política, entendida no como operatoria de poca monta o partidización, sino como apertura a los objetivos que la sociedad y las personas definan. Dicho de otro modo: la ciencia y la técnica son herramientas de la libertad de los ciudadanos y no al revés. Reconstruir la educación pública no es entonces un ejercicio puramente administrativo, centrado en la dependencia de las instituciones, sino que debe ser un proceso político. No hay una finalidad determinada a priori para la educación moderna: ella ha de servirnos según lo que imaginemos y queramos. Esta es la promesa de la educación pública, la humanidad puede autodeterminarse sin tener que seguir a dioses o tutores; educación pública es parte de lo que Kant llamaba la mayoría de edad de nuestra especie, una nueva etapa en su desarrollo abierta por la Ilustración (Kant 1979). Bajo esta lógica, hemos de recuperar la antigua idea de libertad de enseñanza, pero en su sentido político original, no de mercado.

Luego, es necesario que la ciencia se pregunte por la educación. Que intente comprender qué es, cómo sucede, cuáles son sus componentes, etc. Este proceso tampoco ha de tener una finalidad a priori establecida. La imaginación no puede tener ataduras, pues de su libertad depende la capacidad para generar preguntas e hipótesis y también la posibilidad de que se desarrollen múltiples abordajes teóricos y conceptuales para animar un debate científico y pedagógico racional. A pesar de la acumulación histórica de conocimiento de nuestra especie, todavía estamos 
empezando a aprender cómo la vida humana se forma, cómo funciona nuestro cerebro y qué es el lenguaje. Sin límites ni censuras, la ciencia debe abrazar este proceso cuando tiene que ver con la educación, en aras de comprenderlo más cabal y profundamente. Pero el avance de estas preguntas y sus respuestas se ha separado crecientemente de la investigación educativa en Chile (no así en el mundo), centrada en una optimización de organizaciones y desempeños que, a la larga, más agobia que optimiza.

Liberar la imaginación política y la imaginación científica, entonces, supone un primer paso tanto para acrecentar nuestro conocimiento sobre la educación como para deliberar respecto de sus fines. Necesitamos al mismo tiempo más politización y más ciencia. No es una o la otra: son las dos, solo que sin los cerrojos tecnocráticos que, reivindicando el poder de definición política de la evidencia, nos han dejado en estricto rigor sin política ni evidencia (o capacidad de interpretar los hallazgos en un sentido comprensivo). Este ejercicio de ruptura intelectual es necesario si queremos ir más allá del sentido común en esta coyuntura. Si para un niño que es abusado por su padre el deseo espontáneo — de sentido común — es que el maltrato se detenga, imaginar una parentalidad distinta deviene un ejercicio cualitativamente distinto del rechazo inicial al abuso. Es por eso que proponemos revisar el presente desde el futuro antes que desde el pasado.

Si algo debe estar en el centro de las preocupaciones de la educación hoy, eso es el cuidado de la vida. La educación surge como una respuesta ante el hecho de que nuestra vida biológica requiere de la cultura para desarrollarse (Molina, 2015). Ese proceso, aunque puede expresarse luego en habilidades y desempeños, no se limita a ellos. La educación tiene un poder constituyente de vida social e individual, desde las amistades que se forjan en ella hasta el ejercicio de la participación política, pasando por el modo en que racionalizamos y expresamos nuestro mundo interior y nos apropiamos creativamente de la cultura. Todos estos aspectos de la educación tienen por resultado la construcción de soportes para la vida que nos ayudan a construir certezas básicas. En esta hora crítica, la educación debe sostenerlas para proveer seguridad y certezas: mostrar preocupación por lo que pasa con los estudiantes y sus familias, colaborar en la medida de sus capacidades a la tranquilidad de sus hogares, mantener el contacto y las redes de socialización (por la vía que sanitariamente sea posible), etc. Y debe hacerlo a lo largo y ancho de la sociedad, tensándose para llegar a cada rincón de nuestro desigual y fragmentado país.

Una segunda tarea de la educación es vincularse orgánicamente con la respuesta a la crisis. Un ejemplo notable ha sido el esfuerzo de los trabajadores de las universidades públicas - mención destacada merece el personal del Hospital Clínico de la Universidad de Chile- por poner sus laboratorios y demás recursos científicos y tecnológicos al servicio del control de la pandemia. No obstante, este tipo de 
iniciativas más despuntan como excepciones que como norma. Desligar las prácticas de los desempeños estandarizados tradicionales — Simce, acreditación, prosecución normal de estudios, etc.- liberaría tiempo y recursos para que el personal de la educación y sus mismos estudiantes puedan ser parte de la respuesta a la crisis en la medida de sus capacidades. Así, la suspensión y/o flexibilización de las prácticas agobiantes no es un fin en sí mismo, sino solo un medio para que las comunidades generen nuevos objetivos - de alcance nacional y local- relativos al cuidado de la vida y la construcción y soporten espacios de solidaridad tan necesarios en esta hora crítica. Evitar un maltrato intrafamiliar que aumenta a raíz del confinamiento, apoyar las redes de abastecimiento o estimular la creatividad de los estudiantes para contar lo que les ocurre, todas estas son prácticas que ya ocurren en la base de la sociedad y en las tramas de la educación, pero no tienen ni la libertad ni el apoyo para devenir en su papel central. Se trata, además, de prácticas que profesores y profesoras saben vincular con los contenidos de sus materias, al menos en el grado que la actual situación permite. En todos los niveles educativos, la activación de los cursos y las instituciones en torno a estas prácticas va a enriquecer su capacidad de formación disciplinar, académica y pedagógica, no a debilitarla. El país puede confiar con más tranquilidad en sus profesores para este objetivo que en indicadores habituales de desempeño que, ya antes cuestionados, hoy simplemente carecen de sentido a raíz de la coyuntura.

Para que la educación contribuya entonces a dar certezas a la sociedad y proteger el bienestar socioemocional de los chilenos, el Estado debe darle certezas a la educación, lo que pasa por introducir formas provisionales de financiamiento que la vuelvan menos dependiente de la venta unitaria que implican las subvenciones y aranceles, fortaleciendo su capacidad para enfrentar costos fijos. El Estado tiene una responsabilidad con los estudiantes y los trabajadores en cuanto tales; por eso, sin importar dónde estudien, debe asegurar empleos, cancelar o flexibilizar pagos para familias y avanzar decididamente en la condonación de la deuda educativa. Independientemente del lugar en que estudien o trabajen (instituciones estatales o privadas), no hay estudiantes ni trabajadores de primera y segunda categoría. Pero en el caso de la educación pública, además, el Estado tiene una responsabilidad para con ella como proyecto y reservorio cultural, análoga a la que tiene con el mundo del arte y de la cultura mediante otros instrumentos. Debe asegurar más que nunca su funcionamiento, sobrevivencia y proyección.

En el caso de la educación pública, debe haber fondos de emergencia que le permitan enfrentar la pandemia y luego sus efectos económicos. Pero, sobre todo, que le posibiliten dar certezas a las personas, acompañarlas y apoyarlas. Es decir, el sustento a la educación sobrepasa la responsabilidad por sus trabajadores y se proyecta como un factor más (de tipo cultural) de respuesta a la pandemia, como 
ocurre con la enseñanza a través de la televisión y como ha sugerido Unesco y otros organismos internacionales ${ }^{10}$. La educación no es fuente de recursos disponibles para redirigir a salud: ha de ser parte de la respuesta que tanto desde la salud como desde el cuidado se da a la crisis.

En el caso de la educación privada - mayoritaria hoy-, el apoyo estatal debe vincularse a determinados gastos y debe establecer exigencias de cuidado, como obligar a que no haya despidos durante este año y el siguiente, al menos, sin descartar la participación pública en la propiedad. Es decir, lo que debe proteger el apoyo estatal es la educación como respuesta a la crisis y fuente de sociabilidad y empleo, no la solvencia económica de grupos sostenedores o controladores. Se debe apoyar a las instituciones, no a sus dueños. De ahí que insistir en formas sabidamente fracasadas de financiamiento - como créditos blandos a estudiantes- no sea una respuesta a la crisis, sino el mismo comportamiento inercial de las últimas décadas. Todo lo contrario, hoy más que ayer cabe la suspensión del pago de toda deuda educativa controlada por la banca o por el Estado. Y en la medida en que el Estado apoye a las instituciones educativas y cautele que no despidan ni dejen a nadie atrás, estas deben a su vez disminuir los montos y flexibilizar las condiciones del cobro a sus estudiantes, destinando a ello ahorros o liquidez con que sus sostenedores privados cuenten en el sistema financiero. Toda disminución de pagos a la banca o a instituciones se transforma, de manera inmediata y siempre que se haga con responsabilidad, en dinero disponible para consumo, lo que contribuye a la necesaria reactivación de la economía en los próximos meses y a la tranquilidad de las personas y sus familias.

Decíamos al inicio de este artículo que el Covid-19 encuentra un mundo mucho más interconectado que las pandemias que otrora había conocido la historia. Los grados de cooperación humana han aumentado drásticamente en los últimos siglos: cada vez más los individuos de los distintos rincones del planeta se vinculan entre sí, compartiendo productos, ideas y experiencias de otras latitudes como si fueran propias. Este proceso - que ha sido divulgado como globalización - también ha afectado el modo en que la educación y la familia cultivan y desarrollan nuestras vidas. Ni el profesorado ni los padres son ya las figuras exclusivas para la formación de las nuevas generaciones. El hogar y el aula tradicional hoy se ven atravesados cada vez más por relaciones sociales que cruzan fronteras nacionales e identitarias, sea por las nuevas tecnologías o por profundos cambios culturales como aquellos que impulsa el feminismo. Las familias son dinámicas y diversas, y el modo en que los sujetos aprenden de su lugar en la civilización y la historia trasciende la escuela.

10. Ver sitio de Unesco. https://es.unesco.org/covid19/educationresponse 
En este convulso mundo, las personas se tienden a ver a sí mismas como singularidades únicas; el peso de sus familias o de la tradición no tiene ya la fuerza de ayer para definir lo que son o su camino en la vida. Una demanda de mayor autonomía y libertad individual emerge al mismo tiempo que todos dependemos cada vez más de todos. Literalmente, una humanidad más universal despunta en el inicio del siglo XXI, enfrentando como nunca antes - como si se tratara de una sola sociedad sumida en el desconcierto- la pandemia del Covid-19.

Probablemente, esta crisis sanitaria producirá transformaciones que pervivirán en el tiempo. Su tremendo impacto nos recuerda lo precarias que son hoy en día nuestras instituciones de solidaridad y respaldo. Su impacto también nos compele a repensar cómo nuestro poderío tecnológico y económico puede estar al servicio de la vida y no al revés. En lo que a educación y salud respecta, nos ha recordado que la tarea de cultivo de la vida trasciende las burocratizadas y anquilosadas estructuras de desempeño a las que estamos acostumbrados. La salud y la educación no son los mercados de credenciales y de la atención médica que hoy conocemos, sino funciones de creación y desarrollo de la vida que atraviesan a toda la sociedad.

Educación y salud tienen mucho que ver entonces. Son formas conscientes en que cultivamos la vida social e individual y, por lo mismo, tienen que ser vistas como lugares de libertad y no de sometimiento. Hemos de estar atentos a los cambios que dichos procesos han experimentado en las últimas décadas y que hoy, pandemia de por medio, nos miran de frente. A los espacios de derechos sociales como salud y educación no les queda otra opción que repensarse desde sus cimientos. En lo que a educación refiere, no se trata de sumar más capas de control estatal al mercado, sino que, escapando de tales confinamientos políticos e intelectuales, se trata de replantearnos la educación de cara a la manera en que realmente es la vida en el siglo XXI. Debemos enfrentar los desafíos de libertad e igualdad que ayer no fuimos capaces de asumir: es intolerable que hoy en Chile existan dos o más países distintos delimitados por las agudas diferencias sociales y económicas. Los imperativos que nos ha puesto el Covid-19, en realidad, aceleran la necesaria reconstrucción de nuestro sistema educativo y ponen la urgencia de reimaginar la educación pública para el futuro como base de una construcción nacional y moderna todavía pendientes. Apremiada por el Covid-19 y con el proceso constituyente ad portas, la educación chilena ya no puede seguir siendo la misma. 


\section{REFERENCIAS}

Acuña, F. (2015). Incentivos al trabajo profesional docente y su relación con las políticas de evaluación e incentivo económico individual. Estudios Pedagógicos. Vol. 41, pp. 7-26.

Arendt, H. (1997). ¿Qué es la política? Madrid: Paidós.

Bellei, C. (2001). El talón de aquiles de la reforma. Análisis sociológico de la política de los 90 hacia los docentes en Chile. Santiago.

Bellei, C. y Muñoz, G. (2020). La casa no es una escuela: propuestas de política educativa en tiempos de pandemia. Ciper [en línea]. Disponible en: https:// ciperchile.cl/2020/04/08/la-casa-no-es-una-escuela-propuestas-de-politicaeducativa-en-tiempos-de-pandemia/.

Bellei, C., Orellana, V. y Canales, M. (2019). Elección de escuela en la clase alta chilena. Comunidad, identidad y cierre. Archivos Analíticos de Políticas Educativas, vol. (En prensa).

Bellei, C., Valenzuela, J.P., Vanni, X. y Contreras, D. (2014). Lo aprendí en la escuela. ¿Cómo se logran procesos de mejoramiento escolar? [en línea]. S.l.: s.n. ISBN 978956-190-852-9. Disponible en: http://www.uchile.cl/publicaciones/101789/ lo-aprendi-en-la-escuela-como-lograr-procesos-de-mejoramiento-escolar.

Beyer, H. (2001). Entre la autonomía y la intervención: las reformas de la educación en Chile. En: F. Larraín y R. Vergara (eds.), La transformacion económica en Chile. Santiago, Chile: CEP, pp. 644-708.

Bobbio, N. (1989). Estado, gobierno y sociedad. Para una teoría general de la política. México D.F.: FCE.

Brunner, J.J., Elacqua, G., Tillet, A., Bonnefoy, J., González, S., Pacheco, P. y Salazar, F. (2005). Guiar el mercado. Informe sobre la educación superior en Chile. Santiago.

Cox, C. (1994). Las políticas educacionales del gobierno de la transición (Chile, 1990-1994). En Proposiciones, vol. 25, pp. 129-131.

Domedel, A. y Peña y Lillo, M. (2008). El mayo de los pingüinos. Santiago: Radio Universidad de Chile.

Fardella, C. (2016). La universidad managerialista y los dispositivos de gestión del cuerpo académico. Individualización y cuantificación de la experiencia laboral. 2016. Talca: s.n. 
Fardella, C. y Sisto, V. (2015). Nuevas regulaciones del trabajo docente en Chile. Discurso, subjetividad y resistencia. Psicologia \& Sociedade, vol. 27, no. 1, pp. 68-79.

Figueroa, F. (2012). Llegamos para quedarnos. Crónicas de la revuelta estudiantil. Santiago: LOM.

Guajardo, F. y Jara, C. (2018). Educación escolar: cultivando vidas en el mercado educativo. En: V. Orellana (ed.), Entre el mercado gratuito y la educación pública. Dilemas de la educación chilena actual. Santiago: LOM, pp. 157-208.

Huneeus, C. (2016). La reforma universitaria de Bachelet y los intereses políticoeconómicos que la impedirán. El Mostrador.

Kant, I. (1979). Filosofía de la historia. México D.F.: FCE.

Lakatos, I. (1989). La metodología de los programas de investigación cientifica. Madrid: Alianza. ISBN 84-206-2349-0.

Madrid, S. (2016). La formación de masculinidades hegemónicas en la clase dominante: el caso de la sexualidad en los colegios privados de elite en Chile. Sexualidad, Salud y Sociedad (Rio de Janeiro), no. 22, pp. 369-398. DOI 10.1590/1984-6487.sess.2016.22.17.a.

Mizala, A. y Romaguera, P. (2003). El Sistema Nacional de Evaluación del Desempeño Docente (Sned) en Chile. S.l.

Molina, V. (2006). Educación, evolución e individuación. Aproximaciones a una indagación sobre los sentidos de la educación. Revista Prelac, vol. 2, pp. 76-89.

Molina, V. (2015). Más allá de la socialización. Un aporte de las neurociencias a la comprensión del fenómeno educativo. En: M. Morales y H. Burgos (eds.), Descubriendo un cerebro que aprende en el aula. Santiago: Editorial Universidad de Santiago de Chile.

Oecd (2019). Education at a glance 2019 [en línea]. Paris: Oecd. [Consulta: 2 de junio de 2020]. Education at a glance. ISBN 9789264803985. Disponible en: https:// www.oecd-ilibrary.org/education/education-at-a-glance-2019_f8d7880d-en.

Orellana, V. (2011). Nuevos estudiantes y tendencias emergentes en educación superior. Una mirada al Chile del mañana. En: M. Jiménez y F. Lagos (eds.), Nueva geografía de la educación superior. Santiago: Foro Aequalis - Universidad San Sebastián.

Orellana, V., Canales, M., Bellei, C. y Guajardo, F. (2019). Individuación y mercado educacional en Chile. Revista Brasileira de Política e Administração da Educação, vol. 35, no. 1, pp. 141-157. DOI 10.21573/vol1n12019. 
Orellana, V., Miranda, C., Guajardo, F., Sanhueza, J.M., Jara, C. y Carvallo, F. (2018). Entre el mercado gratuito y la educación pública. Dilemas de la educación chilena actual. Santiago: LOM.

Parcerisa, L. y Falabella, A. (2017). The consolidation of the evaluative state through accountability policies: trajectory, enactment and tensions in the chilean education system. Education Policy Analysis Archives, vol. 25, pp. 1-27. ISSN 1068-2341. DOI 10.14507/epaa.25.3177.

Quintela Dávila, G. (2013). Deserción universitaria, una aproximación sociológica al proceso de toma de decisiones de los estudiantes. Sociedad Hoy [en línea]. Vol. 24, no. 1, pp. 83-106. Disponible en: http://www.redalyc.org/articulo. oa?id $=90231580008 \#$.

Redondo, J., Descouvières, C. y Rojas, K. (2004). Equidad y calidad de la educación en Chile. Reflexiones e investigaciones de eficiencia de la educación obligatoria (1990-2001). Santiago: Universidad de Chile- Facso.

Ruiz Encina, C. (2015). De nuevo la sociedad. Santiago: LOM-Fundación Nodo XXI.

Ruiz Encina, C. (2020). Octubre chileno. La irrupción de un nuevo pueblo. Santiago: Taurus.

Ruiz Schneider, C., Reyes, L. y Herrera, F. (2019). Privatización de lo público en el sistema escolar. Chile y la agenda global de educación. Santiago: LOM.

Salazar, J.M. (2015). Leyendo vísceras: la reforma a la educación superior en perspectiva. Cuadernos de Coyuntura Nodo XXI, vol. Año 3, no. 10, pp. 28-41. ISSN 0124-3543.

Salazar, J.M. y Leihy, P.S. (2013). El manual invisible: Tres décadas de políticas de educación superior en Chile (1980-2010). Archivos Analíticos de Políticas Educativas, vol. 21, no. 34.

Sanhueza, J.M. y Orellana, V. (2018). Análisis crítico de la reforma educacional de Bachelet (2014-2018). En: V. Orellana (ed.), Entre el mercado gratuito y la educación pública. Dilemas de la educación chilena actual. Santiago: LOM, pp. 259-322.

Varios autores (2020). Propuestas educación. Trabajo interuniversitario, Mesa Social Covid-19. Santiago.

Weber, M. (2005). El político y el científico [en línea]. Madrid: Alianza. Disponible en: http://www.hacer.org/pdf/WEBER.pdf. 